\title{
Comparación de la Escala de RIPASA y Alvarado Modificada en la determinación de Apendicetomía a través de Curvas ROC
}

\section{Comparison of the RIPASA and Modified Alvarado Scale in the determination of Appendicetomy through ROC Curves}

\author{
Jessica Alexandra Marcatoma Tixi. ${ }^{1}$, Héctor Salomón Mullo Guaminga. ${ }^{2}$, Natalia \\ Alexandra Pérez Londo. ${ }^{3}$ \& Mayra Yolanda Almache Caiza. ${ }^{4}$
}

Recibido: 20-02-2021 / Revisado: 29-02-2021 /Aceptado: 21-03-2021/ Publicado: 05-04-2021 
study (phlegmonous appendicitis), among the main symptoms that help to diagnose appendicitis are migratory pain $(71.5 \%)$ and pain in the right iliac fossa $(98 \%)$. Regarding the analysis of the Modified Alvarado scale and RIPASA, it is obtained that the Alvarado scale through the ROC curve has an area (0.583), sensitivity (69.78\%), specificity $(82.78 \%)$, true positive fraction $(65.76 \%)$, true negative fraction $(20 \%)$, contrary to RIPASA with one area (0.594), sensitivity (88.4\%), specificity (90.9\%), true positive fraction $(68.90 \%)$, true negative fraction $(25.58 \%)$. Conclution. It is concluded that the RIPASA scale presents greater certainty when diagnosing acute appendicitis and its use is recommended in the Emergency Services.

Keywords: Appendicitis, ROC curves, sensitivity, specificity.

\section{Resumen.}

Introducción. La apendicitis aguda es una enfermedad repentina que ocurre en cualquier etapa de la vida, es la primera causa de atención quirúrgica en el servicio de emergencia de todos los hospitales y entre los tratamientos se encuentra la apendicectomía Objetivo. Identificar la escala más robusta entre Alvarado Modificada y la escala de RIPASA para discriminar la necesidad de cirugía en pacientes diagnosticados con apendicitis aguda. Metodología. El diseño de investigación fue exploratoria con información recolectada en el período junio 2010 - enero 2019, la matriz de datos compiló información de 400 historias clínicas de pacientes atendidos en el servicio de emergencia del Hospital General Docente Riobamba con dicha anomalía, considerando 18 variables; 5 de tipo cuantitativo y 13 mudables estadísticas, previo al análisis se realizó imputación de datos faltantes, con la ayuda de la moda para mudables y mediante regresión para variables cuantitativas. Resultados. De los pacientes apendicectomizados el 50.8\% corresponden a hombres y el $49.3 \%$ a mujeres, por otro lado, el $50 \%$ de los pacientes poseen una edad menor o igual a los 24 años. El Grado de apendicitis II es el más usual en el estudio (Apendicitis flegmonosa), entre los principales síntomas que ayudan a un diagnóstico de apendicitis son dolor migratorio (71.5\%) y el dolor en la fosa ilíaca derecha (98\%). Con respecto al análisis de la escala de Alvarado Modificada y RIPASA, se obtienen que la escala de Alvarado mediante la curva ROC posee un área (0.583), sensibilidad (69.78\%), especificidad (82.78\%), fracción verdaderos positivos $(65.76 \%)$, fracción verdaderos negativos (20\%), al contrario de RIPASA con un área (0.594), sensibilidad (88.4\%), especificidad (90.9\%), fracción verdaderos positivos $(68.90 \%)$, fracción verdaderos negativos (25.58\%). Conclusión. Se concluye que la escala de RIPASA presenta mayor certeza al momento de diagnosticar apendicitis aguda y se recomienda su uso en los Servicios de Emergencia.

Palabras claves: Apendicitis, Curvas ROC, sensibilidad, especificidad, 


\section{Introducción.}

La apendicitis aguda es una enfermedad súbita y repentina que puede ocurrir en cualquier etapa de la vida, (Wani, y otros, 2007) es la primera causa de atención quirúrgica en el servicio de emergencia de todos los hospitales y entre los tratamientos de oro para su recuperación se encuentra la apendicectomía, indicación de urgencias más frecuente dentro de la medicina contemporánea. (Fernández Zambrano, 2016) (Fernández, 2016)

La presencia de esta patología se observa en 1 de cada 10 individuos a lo largo de la vida (Ávila \& García-Acero, 2015) siendo más frecuente entre los hombres con un $8.6 \%$ en comparación con el $6.7 \%$ de las mujeres, (Martin \& Stella K, 2018) su mayor incidencia se alcanza entre los 20 y 35 años, pero el riesgo para desarrollarla no desaparece en otras edades, e inclusive es más alta en el quinquenio de 10 a 19 años, no obstante se reporta que uno de cada 2000 adultos mayores de 65 años desarrollará apendicitis anualmente. (Zambrano, Ramos, \& Merino, 2019) Su importancia de saneamiento radica en el desarrollo de complicaciones como formación de flemón o abscesos (Smink \& Soybel, 2019) por lo que su diagnóstico y tratamiento debe ocurrir en las primeras etapas para evitar inconvenientes.

El diagnóstico de la AA se basa principalmente en los hallazgos clínicos, difícil, en especial, en las primeras horas del cuadro clínico, (Humes \& Simpson, 2012) por lo que la determinación definitiva de necesidad de cirugía sigue siendo un reto, por un lado debido a la diversidad de métodos y técnicas que utilizan los cirujanos para decidir si ejecutarla o no y por otro, a la variedad de síntomas que pueden presentar los pacientes cuando acuden al centro de salud con dolor abdominal, más aún cuando se conoce que un cirujano experto y con práctica habitual en un servicio de urgencias puede tener hasta un $15 \%$ de apendicectomías negativas, pero esta cifra puede incrementarse, en especial en mujeres menores de 35 años, hasta el 26\%. (Humes \& Simpson, 2012)

Varias son las técnicas de diagnóstico para la enfermedad y aun el área de salud continúa en la definición de una metodología significativa en cuanto a patrones de sensibilidad y especificidad, los métodos que hasta el momento se manejan todavía arrojan porcentajes elevados de falta de asertividad en el requerimiento de cirugía frente a tan solo un tratamiento clínico en los pacientes, por ejemplo en Estados Unidos el número de tomografías ante la sospecha de AA en adultos, de 18.5\% de los casos en 1998 se elevó a $93.2 \%$ en el 2007. Las apendicectomías negativas en mujeres menores de 45 años de edad se redujeron de $42.9 \%$ en 1998 a $7.1 \%$ en 2007. Sin embargo, esto no sucedió en la población masculina, ni en mujeres mayores de 45 años, a pesar de la tomografía preoperatoria. (Coursey, y otros, 2010) En cuanto a los hallazgos en los exámenes de laboratorio, ninguna de estas pruebas confirma o excluye el diagnóstico de apendicitis aguda cuando se utilizan de manera aislada, ya sean las alteraciones leucocitarias (leucocitosis $87 \%$ o leucopenia $10 \%$ de los casos), proteína C reactiva, o marcadores nuevos como lactoferrina, calprotectina, d-lactato, etc. (Thuijls, y otros, 2011) (Laméris, y otros, 2009) (Filiz, y otros, 2010) 
Con tales antecedentes optar por una técnica de diagnóstico sensible y específica ante la sintomatología de los pacientes deja de lado a los métodos tradicionales. En la actualidad existen dos métodos de valoración cuasi cuantitativa, la escala de Alvarado modificada considerada como la de mayor difusión y aceptación en los servicios de urgencias del mundo, su sensibilidad y especificidad (Reyes-García, y otros, 2012) es de 68\% de 87.9\% respectivamente, clasifica a los pacientes en 3 grupos luego de la valoración de signos, síntomas y exámenes de laboratorio: Riesgo bajo (0-4 puntos) cuya probabilidad de presentar apendicitis es $7.7 \%$ y el tratamiento es netamente ambulatorio, Riesgo medio (5-7 puntos) asocia un $56.7 \%$ de presencia de apendicitis y requiere hospitalización y Riesgo alto (8-10 puntos) con una probabilidad de $90.6 \%$ de apendicitis, estos pacientes deben ser sometidos a cirugía inmediata (Reyes-García, y otros, 2012) y la escala de RIPASA cuya sensibilidad y especificidad es de $98 \%$ y $83 \%$ respectivamente, de igual manera agrupa a los pacientes luego de la valoración de características sociodemográficas, signos, síntomas y exámenes de laboratorio en: Improbable con una puntuación inferior a 5 puntos, Baja probabilidad entre 5 y 7 puntos y Alta probabilidad entre 7.5 y 11.5 puntos por tanto la preparación para una apendicetomía es inmediata. (Chong, y otros, 2011) (Klabtawee, Saensak, Khetsoongnern, \& Piriyasupong, 2011)

El objetivo de la investigación fue evaluar el sistema de diagnóstico robusto entre la escala de Alvarado modificada y la escala de RIPASA para discriminar la necesidad de cirugía en pacientes diagnosticados de apendicitis aguda en el servicio de emergencia de una casa de salud en Riobamba, junio 2010 - enero 2019 en torno a los porcentajes de sensibilidad y especificidad. (Almache Caiza \& Mena Chavarrea, 2019)

\section{Metodologia.}

Por el método de investigación se consideró mixta ya que la información se concentró en mudables y variables estadísticas; según el objetivo fue aplicada ya que se procedió a solucionar un problema específico en el área de salud; con respecto al nivel de profundización en el objeto de estudio fue considerada exploratoria debido al estudio general de comportamiento entre la escala de Alvarado Modificada y la escala de RIPASA al momento de discriminar la necesidad de cirugía en pacientes diagnosticados de apendicitis aguda. (Almache Caiza \& Mena Chavarrea, 2019)

\section{Instrumentos de recolección de datos}

Las historias clínicas fueron los instrumentos donde reposaba la información de los pacientes diagnosticados de apendicitis aguda, conforme se leían los datos de los pacientes se seleccionó 18 variables de las cuales 13 son cualitativas (estado civil, sexo, náusea/vómito, anorexia, tipo de apendicitis, dolor migratorio, tipo de dolor, dolor fosa ilíaca derecha, signo de Bloomberg, signo de Rovsing, resistencia muscular voluntaria, hipersensibilidad en la fosa ilíaca derecha y examen de orina) y 5 variables de índole cuantitativo (edad, temperatura, evolución con el síntoma, leucocitos y neutrófilos). (Almache Caiza \& Mena Chavarrea, 2019) 


\section{Población y Muestra}

Se trabajó con un colectivo de 400 pacientes diagnosticados con apendicitis aguda del Hospital General Docente de Riobamba quienes al derivarse de un marco muestral no definido se constituyeron como la muestra del estudio.

\section{Técnicas estadísticas}

El análisis exploratorio de datos permitió describir las características generales de los pacientes, para el análisis bivariado se utilizó tablas de contingencia que indicaron la dependencia o no de variables, dentro de las técnicas multivariadas se destaca las Curvas ROC mismas que permitieron visualizar, organizar y seleccionar el requerimiento de cirugía en pacientes con apendicitis aguda.

El análisis de datos se desarrolló en el lenguaje de programación R.

\section{Resultados.}

Durante el período comprendido entre Junio 2010 a Enero 2019 se evaluó 400 pacientes que fueron atendidos con apendicitis, en el servicio de emergencia del Hospital General Docente de Riobamba, considerando 18 variables; 5 de tipo cuantitativo (Neutrófilos, Temperatura, Evolución con el síntoma, Leucocitos y Edad), y 13 mudables estadísticas (Estado civil, Sexo, Nauseas/Vómito, Anorexia, Grado de apendicitis, Dolor Migratorio, Tipo de dolor, Dolor en la fosa iliaca derecha, Signo de Bloomberg, Signo de Bloomberg, Resistencia Muscular, Hipersensibilidad en la fosa iliaca derecha, Examen de Orina ). (Almache Caiza \& Mena Chavarrea, 2019)

\begin{tabular}{ccc}
\hline Sexo & $\mathbf{n}_{\mathbf{i}}$ & $\boldsymbol{\%}$ \\
\hline Femenino & 197 & 49.3 \\
Masculino & 203 & 50.8 \\
Total & 400 & 100 \\
\hline
\end{tabular}

Tabla 1.Distribución estadística de la variable sexo

Fuente: Elaboración propia

La muestra obtenida, estuvo conformada por 197 mujeres (49.3\%) y 203 hombres (50.8\%) valorados con un cuadro de dolor abdominal y sospecha de apendicitis, esta patología fue mayor en los hombres (Tabla 1). De los pacientes atendidos el de menor edad tenía 2 años y el mayor 85 años, con una población predominante joven, con una edad media de $29 \pm 17$ años (Díaz-Barrientos C. , y otros, 2018) además el 50\% de los pacientes poseían una edad menor o igual a los 24 años. (Almache Caiza \& Mena Chavarrea, 2019) 


\begin{tabular}{lccccc}
\hline & Edad & Temperatura & $\begin{array}{c}\text { Evolución } \\
\text { con el } \\
\text { síntoma }\end{array}$ & Leucocitos & Neutrofilia \\
& 29 & 36.98 & 36 & 14245.292 & 75.60 \\
Media & 24 & 36.9 & 24 & 13550 & 78.45 \\
Mediana & 18 & 36 & 24 & 15600 & 84 \\
Meda & 17 & 0.94 & 48 & 10123.25 & 12.54 \\
Mínimo & 2 & 34.2 & 1 & 1480 & 11.62 \\
Máximo & 85 & 40.5 & 720 & 151000 & 97.39 \\
\hline
\end{tabular}

Tabla 2. Estadísticas descriptivas de las variables cuantitativas

Fuente: Elaboración propia

La temperatura mínima de los pacientes fue de $34.2^{\circ} \mathrm{C}$, el cual mostró un cuadro de hipotermia que generalmente manifiestan los adultos mayores, la temperatura máxima fue de $40.5^{\circ} \mathrm{C}$ indicó la presencia de fiebre, en promedio la temperatura fue de $36.98 \pm$ $0.94{ }^{\circ} \mathrm{C}$, el $50 \%$ de las personas conservaron una temperatura menor o igual $36.9^{\circ} \mathrm{C}$. La evolución con el síntoma es propia de cada enfermedad, en el caso estudiado el mínimo de horas de evolución de un paciente reiteró 1 hora de dolor y su máximo fue un total de 720 horas (30 días), en promedio la evolución con el síntoma se englobó en $36 \pm 48$ horas, el $50 \%$ de los pacientes presentaron dicha característica en un tiempo menor o igual a 24 horas; la mayoría de los pacientes soportaron el síntoma por 24 horas antes de recibir los primeros auxilios (triage). (Almache Caiza \& Mena Chavarrea, 2019) El recuento de leucocitos se refiere al número de glóbulos blancos que se explora en la sangre de cada paciente, el número mínimo de leucocitos que se examinó fue de 1.480 células/mm3, mientras que el máximo punteó un valor de 151.000 células $/ \mathrm{mm} 3$, en promedio estas células se mantuvieron en un total de $14.245 \pm 10.123$ células $/ \mathrm{mm} 3$, el $50 \%$ de los glóbulos blancos asociaron un valor menor o igual a 13.550 células/mm3; la mayoría de los pacientes asumieron un conteo de 15.600 células $/ \mathrm{mm}^{3}$. (Almache Caiza \& Mena Chavarrea, 2019) Finalmente, dentro de las células defensivas, los neutrófilos son los primeros en llegar al tejido afectado, seguidos por los macrófagos y linfocitos cuando son afectados con algún tipo de patología. El valor mínimo de neutrófilos era de $11.62 \%$, con un máximo de $97.39 \%$, en promedio los neutrófilos tuvieron un valor aproximado de $75.60 \%$, el $50 \%$ de las células son menor o igual a $78.45 \%$; la mayor cantidad de neutrófilos que relucieron los pacientes es de $84 \%$ con una variación del $12.54 \%$. (Almache Caiza \& Mena Chavarrea, 2019)

\begin{tabular}{ccccc}
\hline & \multicolumn{2}{c}{ Nauseas o Vómito } & \multicolumn{2}{c}{ Anorexia } \\
& $\mathbf{n}_{\mathbf{i}}$ & $\mathbf{\%}$ & $\mathbf{n}_{\mathbf{i}}$ & $\boldsymbol{\%}$ \\
\hline $\mathrm{Si}$ & 280 & 70 & 18 & 95.5 \\
No & 120 & 30 & 382 & 4.5 \\
Total & 400 & 100 & 400 & 100 \\
\hline
\end{tabular}

Tabla 3. Distribución estadística de Nauseas o Vómito y Anorexia

Fuente: Elaboración propia 
Los primeros síntomas de apendicitis son de vital importancia; mientras más temprano se conozca la anomalía, menor será la posibilidad de evolucionar de una forma grave. (Ferreira, 2019) Para la obtención del diagnóstico de Apendicitis uno de los síntomas que ayuda son las Nauseas o Vómito, la sintomatología suele aparecer después del dolor abdominal, siendo un indicio inespecífico y fácil de confundirlo como síntoma de otra anomalía (Thompson, 2012) (Díaz-Barrientos C. Z., y otros, 2018). De los 400 casos intervenidos el $70 \%$ de los pacientes presentaron náuseas o vómito, los cuales el $4.5 \%$ mostraron la presencia de Anorexia (Tabla 3.). Además, se presentó una diferencia del $34.8 \%$ (139 pacientes) entre grado II o flegmonosa y Grado III o gangrenosa, mientras que de forma uniforme se encuentró el Grado I o Simple y Grado IV o Perforada en pacientes con problemas en el apéndice (Tabla 4.).

\begin{tabular}{ccc}
\hline Grado de Apendicitis & $\mathbf{n}_{\mathbf{i}}$ & $\boldsymbol{\%}$ \\
\hline Grado I & 59 & 14.8 \\
Grado II & 219 & 54.8 \\
Grado III & 80 & 20 \\
Grado IV & 42 & 10.5 \\
Total & 400 & 100
\end{tabular}

Tabla 4. Distribución estadística de Grado de Apendicitis

Fuente: Elaboración propia

\begin{tabular}{ccc}
\hline Dolor Migratorio & $\mathbf{n}_{\mathbf{i}}$ & $\boldsymbol{\%}$ \\
\hline $\mathrm{Si}$ & 286 & 71.5 \\
$\mathrm{No}$ & 114 & 28.5 \\
Total & 400 & 100 \\
\hline
\end{tabular}

Tabla 5. Distribución estadística de Dolor Migratorio

Fuente: Elaboración propia

Al evaluar el resto de los síntomas el $71.5 \%$ de los pacientes presentó dolor migratorio (Tabla 5.), sin embargo, el tipo de dolor que manifestaron los pacientes con problemas de apendicitis fue moderado con un $68.3 \%$, de manera similar muestran una diferencia del $42.3 \%$ (169 pacientes) en relación a los pacientes que manifestaron dolor intenso (Tabla 6.).

\begin{tabular}{ccc}
\hline Tipo de Dolor & $\mathbf{n}_{\mathbf{i}}$ & $\boldsymbol{\%}$ \\
\hline Intenso & 104 & 26 \\
Leve & 23 & 5.8 \\
Moderado & 273 & 68.3 \\
Total & 400 & 100 \\
\hline
\end{tabular}

Tabla 6. Distribución estadística de Tipo de Dolor

Fuente: Elaboración propia

De la misma forma uno de los síntomas presentados con frecuencia de la apendicitis aguda es el dolor abdominal presente a nivel de la fosa ilíaca derecha con mayor o menor 
intensidad. (Montero Tapia, 2016) Por consiguiente el $98 \%$ de los pacientes presentaron dicho dolor, mientras que el $2 \%$ no muestran indicios del malestar (Tabla 7.), en cambio el signo de Bloomberg suele manifestarse como dolor cuando se realiza una descompresión brusca de la pared abdominal y que puede ser referido con mayor intensidad sobre la fosa ilíaca derecha (Serrano Serrano , 2016), el cual se mostró en el 93\% de pacientes al finalizar el examen físico (Tabla 7.); por otra parte, el síntoma signo de Rovsing (Tabla 7.) presentó el $75 \%$ de los pacientes y $25 \%$ no evidenció dicha característica. Con respecto a la resistencia muscular el $91 \%$ de los pacientes no mostró evidencia de dicha particularidad, mientras que el $9 \%$ manifestaron esta particularidad (Tabla 8.).

\begin{tabular}{ccccccc}
\hline \multirow{2}{*}{ Casos } & \multicolumn{2}{c}{$\begin{array}{c}\text { Dolor en la fosa ilíaca } \\
\text { derecha }\end{array}$} & \multicolumn{2}{c}{ Signo de Bloomberg } & \multicolumn{2}{c}{ Signo de Rovsing } \\
\cline { 2 - 7 } & $\mathbf{n}_{\mathbf{i}}$ & $\boldsymbol{\%}$ & $\mathbf{n}_{\mathbf{i}}$ & $\boldsymbol{\%}$ & $\mathbf{n}_{\mathbf{i}}$ & $\boldsymbol{\%}$ \\
\hline $\mathrm{Si}$ & 392 & 98 & 373 & 93.25 & 299 & 74.75 \\
$\mathrm{No}$ & 8 & 2 & 27 & 6.75 & 101 & 25.25 \\
Total & 400 & 100 & 400 & 100 & 400 & 100 \\
\hline
\end{tabular}

Tabla 7. Distribución estadística del Dolor en la fosa ilíaca derecha, Signo de Bloomberg y Signo de Rovsing

Fuente: Elaboración propia

\begin{tabular}{ccccc}
\hline \multirow{2}{*}{ Casos } & \multicolumn{2}{c}{ Resistencia Muscular } & \multicolumn{2}{c}{$\begin{array}{c}\text { Hipersensibilidad en } \\
\text { la fosa ilíaca derecha }\end{array}$} \\
\cline { 2 - 5 } & $\mathbf{n}_{\mathbf{i}}$ & $\boldsymbol{\%}$ & $\mathbf{n}_{\mathbf{i}}$ & $\boldsymbol{\%}$ \\
\hline $\mathrm{Si}$ & 35 & 8,75 & 391 & 97,75 \\
$\mathrm{No}$ & 365 & 91,25 & 9 & 2,25 \\
Total & 400 & 100 & 400 & 100 \\
\hline
\end{tabular}

Tabla 8. Distribución estadística de Resistencia Muscular e Hipersensibilidad en la fosa ilíaca derecha

Fuente: Elaboración propia

La hipersensibilidad de la fosa ilíaca derecha o signo de Mc Burney, se manifiesta al presionar la fosa ilíaca derecha en un punto que corresponde a la unión del 1/3 externo con los 2/3 internos de una línea trazada de la espina ilíaca anterosuperior derecha hasta el ombligo. (Orbea Marcial, 2012) Por esta razón, es la más frecuente con el $98 \%$ en pacientes diagnosticados con apendicitis (Tabla 8.). Al aplicar el examen de orina el $85 \%$ de los pacientes no presentaron infección urinaria, su el diagnóstico fue negativo (Tabla 9.).

\begin{tabular}{ccc}
\hline Casos & $\boldsymbol{n}_{\boldsymbol{i}}$ & $\boldsymbol{\%}$ \\
\hline Negativo & 340 & 85 \\
Positivo & 60 & 15 \\
Total & 400 & 100 \\
\hline
\end{tabular}

Tabla 9. Distribución estadística de frecuencia de la mudable Examen de orina3

Fuente: Elaboración propia 
Según los indicadores sociodemográficos se analizó la prueba de masculinidad de los pacientes con problemas de apendicitis, teniendo así que de cada 100 mujeres que presentaron esta patología 103 hombres lo mostraron. (Almache Caiza \& Mena Chavarrea, 2019)

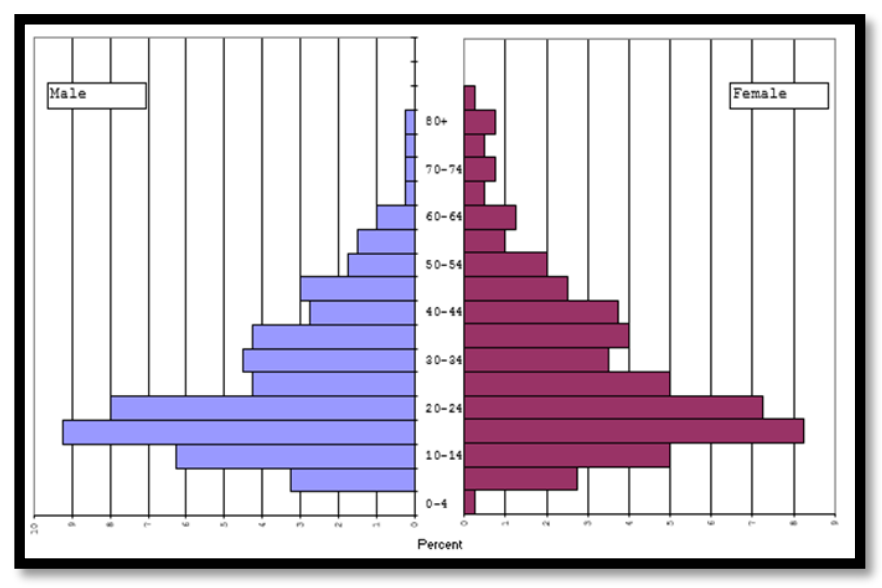

Figura 1. Pirámide Poblacional Fuente: Elaboración propia

Según la pirámide poblacional (Figura 1.) existió la presencia de una pirámide estacionaria con una base más estrecha y un número de personas aproximadamente igual en cada grupo de edades, generalmente ilustró una proporción moderada de niños y una tasa de crecimiento lenta ya que la mayor concentración se localizó entre las edades de 15 a 24 años tanto en hombres como mujeres, es decir existió más población de adolescentes que presentaron problemas de apendicitis. (Almache Caiza \& Mena Chavarrea, 2019)

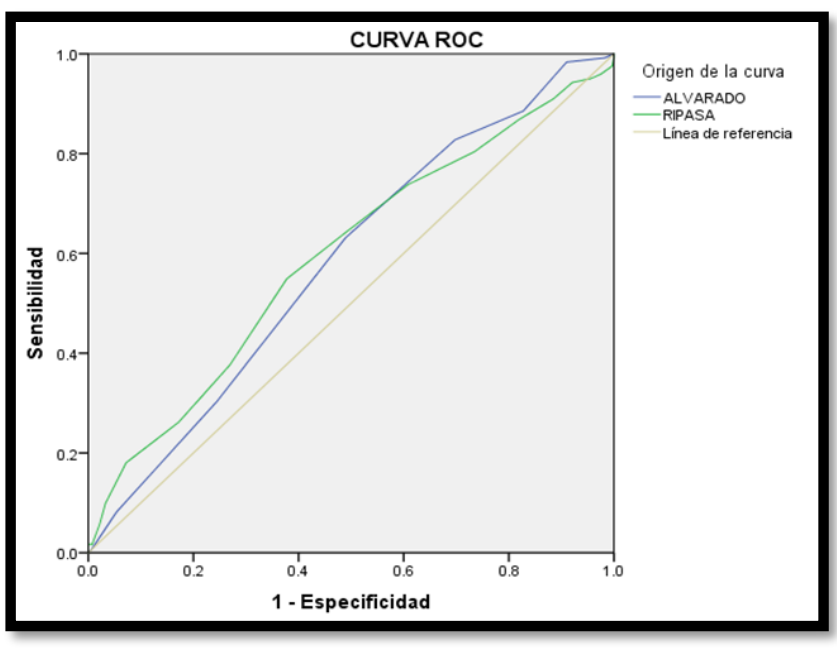

Figura 2. Curva ROC comparativa de la escala de Alvarado modificada y la escala de RIPASA Fuente: Elaboración propia

Para evaluar el sistema de diagnóstico robusto entre la escala de Alvarado modificada y la escala de RIPASA, (Almache Caiza \& Mena Chavarrea, 2019) se realizó los cálculos 
de la curva ROC, sensibilidad, especificidad, fracción de verdaderos positivos (FVP) y fracción de verdaderos negativos (FVN).

\begin{tabular}{cccc}
\hline \multirow{2}{*}{ ALVARADO } & \multicolumn{2}{c}{ ESTADO DEL } & \\
\cline { 2 - 3 } & \multicolumn{2}{c}{ PACIENTE } & Total \\
\cline { 2 - 3 } & CRÓNICO & AGUDO & \\
\hline 7 & 194 & 101 & 295 \\
Total & 84 & 21 & 105 \\
& 278 & 122 & 400 \\
\hline
\end{tabular}

Se generó las curvas ROC para las dos escalas, donde, la línea de referencia indicó teóricamente que las escalas serían incapaces de discriminar a los pacientes agudos de los pacientes crónicos, con una probabilidad entre 0.5 y 0.6 , sin embargo, ambas escalas poseen igual sensibilidad y especificidad (Figura 2.). El 58.3\% representó el área cubierta de la escala de Alvarado Modificada, mientras que el 59.4\% conformó el área de la Escala de RIPASA; por lo que se estableció una diferencia porcentual del $1.1 \%$; lo que significa que existió cierta homogeneidad entre ellas, por lo tanto, la escala de RIPASA fue superior con respecto a la de Alvarado.

Tabla 10. Tabla de contingencia Escala Alvarado Modificada

Fuente: Elaboración propia

\begin{tabular}{cccc}
\hline \multirow{2}{*}{ RIPASA } & \multicolumn{2}{c}{ ESTADO DEL } & \multirow{2}{*}{ Total } \\
\cline { 2 - 3 } & \multicolumn{2}{c}{ PACIENTE } & \\
\cline { 2 - 3 }$>7.5$ & 246 & 111 & 357 \\
$<7.5$ & 32 & 11 & 43 \\
Total & 278 & 122 & 400 \\
\hline
\end{tabular}

Tabla 11. Tabla de contingencia Escala RIPASA

Fuente: Elaboración propia

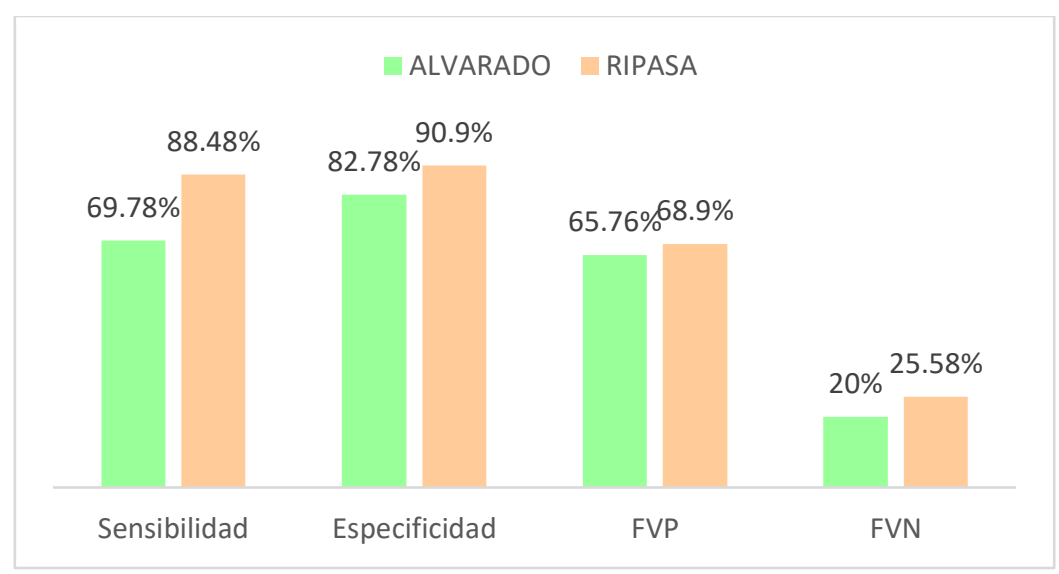

Figura 3. Resultado de Resultados de sensibilidad, especificidad, fracción de verdaderos positivos (FVP) y fracción de verdaderos negativos (FVN)

Fuente: Elaboración propia

Mediante tablas de contingencia se estableció puntos de corte para el estado con el que ingresó el paciente (crónico, agudo) (Almache Caiza \& Mena Chavarrea, 2019)en ambas escalas. En la Escala de Alvarado se determinó un punto de corte de 7 (Tabla 10), donde se obtuvo 295 pacientes con un diagnóstico confirmado de apendicitis superior a los 7 puntos, por lo que representó una buena sensibilidad de $69.78 \%$, especificidad de $82.78 \%$ 
y un FVP de $65.76 \%$ (Figura 3). De la misma forma se estableció un punto de corte de 7.5 para la escala de RIPASA (Tabla 11), donde se obtuvo 357 pacientes con un diagnóstico confirmado de apendicitis superior a los 7.5 puntos, teniendo esto una sensibilidad de $88.48 \%$, especificidad de $90.9 \%$ y un FVP de $68.9 \%$ (Figura 3). Por lo tanto, la escala de RIPASA tuvo mayor exactitud al diagnosticar apendicitis, realizando un mejor tamizaje de los pacientes con dicha patología. (Almache Caiza \& Mena Chavarrea, 2019)

\section{Discusión}

La exactitud de $75 \%$ a $90 \%$ del diagnóstico de apendicitis aguda se establece por la historia clínica y la exploración física. Sin embargo, el estudio debe respaldarse (DíazBarrientos C. Z., y otros, 2018) con estudios paraclínicos. La mujer debe ser sometida a un examen pélvico y el hombre debe realizarse un tacto renal para la exploración física completa del abdomen. El diagnostico incorrecto o tardío aumenta el riesgo de complicaciones como infección de la herida quirúrgica ( $8 \%$ a15\%), perforación del apéndice (5\% a 40\%), abscesos (2\% a 6\%), sepsis y muerte (0.5\% a $5 \%$ ). (DíazBarrientos, y otros, 2018) (Wani, y otros, 2007) (Shrivastava, Gupta, \& Sharma, 2004)

En este estudio la población predominantemente fue joven con promedio de edad de $29 \pm$ 17 años, similar a lo encontrado por Chong C. et al. (2017) (Guallpa Guallpa, 2019) el cual determino una edad promedio más joven de $29.5 \pm 13.3$ años (Chong, y otros, 2017). Malik M. et al. (2017), en una población oriental encontró una edad promedio de $22.7 \pm$ 9.2 años. (Malik, y otros, 2017)

El diagnóstico oportuno y certero de apendicitis resulta fundamentalmente importante para este estudio el cual concuerda a estudios previos realizados en España, México, Cuba, Colombia, Perú y Ecuador, donde la molestia en la apéndice se presentó con mayor frecuencia en los hombres, así también, concuerda con el estudio de Serrano Tatiana (2016), el cual analizó a 151 pacientes y obtuvo como resultado que, la mayoría de pacientes que presentaron un malestar (Almache Caiza \& Mena Chavarrea, 2019) en el apéndice, fueron de sexo masculino con un $64.20 \%$ y el $35.80 \%$ fueron de sexo femenino. (Serrano Serrano T. C., 2016). Mendoza J. (2010), en su investigación obtuvo en cuanto al sexo, el diagnostico de apendicitis con mayor frecuencia fue en los hombres con 55.8\% el en relación a las mujeres (44.2\%). (Mendoza, Rodríguez, \& Guerrero, 2010) De igual manera Thompson Natalia (2012) en el Centro Médico Naval encontró como resultado que el $65.5 \%$ de los pacientes con problemas en el apéndice fueron hombres y $34.5 \%$, fueron mujeres; por lo tanto, el sexo que predominó fue el masculino. (Thompson, 2012)

Además de los 400 casos intervenidos, el $70 \%$ de los pacientes presentaron náuseas o vómito, lo que coincide con el trabajo de Orbea Víctor (2012), donde la población en estudio fue de 194 pacientes atendidos en el Hospital Provincial Puyo y el 86.59\% de los pacientes tuvo nauseas o vómito (Almache Caiza \& Mena Chavarrea, 2019) (Orbea Marcial, 2012). De esta manera se obtuvo también que el $4.5 \%$ de los pacientes 
presentaron anorexia el cual está sustentado por la investigación de Orbea Víctor al realizar un análisis en 194 pacientes atendidos en el Hospital Provincial Puyo (Almache Caiza \& Mena Chavarrea, 2019), donde se evidencio que el 51.54\% tenían Anorexia. (Orbea Marcial, 2012)

La investigación realizada por Thompson Natalia (2012), en el Centro Médico Naval, obtuvo como resultado que el $73.3 \%$ de los pacientes presentaron Náuseas/Vómitos y el 66.4\% manifestaron Anorexia. (Thompson, 2012) Sanabria Á. (2010), indicó que el 79.7\% de pacientes tuvieron Náuseas/Vómitos y 63\% Anorexia. (Sanabria, Mora, Domínguez, Vega, \& Osorio, 2010). Sin embargo, el autor De Quesada L. (2015), mostró que el $80 \%$ de los pacientes padecieron de Náuseas/Vómitos y el 70\% de Anorexia. (de Quesada Suárez, Ival Pelayo, \& González Meriño, 2015)

El análisis realizado por Orbea Víctor (2012), en el Hospital Provincial Puyo, mostró que el $50 \%$ de los portadores de la enfermedad fueron de grado II y el $30.9 \%$ de las personas atendidas presentaron un grado III (Almache Caiza \& Mena Chavarrea, 2019), coincidiendo con nuestros resultados los cuales el 54.8\% de los pacientes con problemas en el apéndice fue de grado II y el $20 \%$ fue de Grado III. (Orbea Marcial, 2012) Por otra parte, el $71.5 \%$ de los pacientes mostraron dolor migratorio, el cual concuerda con el autor Orbea Víctor (2012), que, en su estudio, el 60.3\% de los pacientes tuvieron (Almache Caiza \& Mena Chavarrea, 2019) la presencia de dolor migratorio. (Orbea Marcial, 2012) De forma similar el estudio de Thomson Natalia (2012), en el Centro Médico Naval mostró que el $81.7 \%$ de los pacientes manifestaron dolor migratorio. (Thompson, 2012) Así mismo, en la investigación de Sanabria Álvaro et al. (2010), se observó, que el 66.7\% de los casos tuvo dolor migratorio. (Sanabria, Mora, Domínguez, Vega, \& Osorio, 2010) En el trabajo de López Abreu et al. (2016), se concluye que el $84 \%$ de los pacientes presentaron dolor migratorio. (López Abreu, Fernández Gómez, Hernández Paneque, \& Pérez Suárez, 2016)

Se generaron las curvas ROC para las escalas, donde se observó, el área bajo la curva de 0,583 para la escala de Alvarado Modificado y 0.594 para la escala de RIPASA, siendo superior la escala de RIPASA. Estos resultados se contrastan con lo encontrado por C.Z.Díaz-Barrientos (2018), que muestran en la escala de Alvarado un área de 0.719 superior a la escala de RIPASA (AUC de 0.595). (Díaz-Barrientos C. Z., y otros, 2018) Del mismo modo Golden et al. (2016), en EEUU obtuvo un área bajo la Curva ROC de 0.67 para la escala de RIPASA y 0.72 para la escala Alvarado Modificado, esto puede deberse a que la escala de RIPASA fue diseñada para población asiática. (Arroyo-Rangel, Limon, Vera, Guardiola, \& Sánchez-Valdivieso, 2018) (Golden , y otros, 2016) Sin enbargo, Arroyo Rangel et al. (2018), indicó una AUC de 0.88 para la escala de RIPASA similar en comparación con la escala de Alvarado (AUC de 0.8). (Arroyo-Rangel, Limón, Vera, Guardiola, \& Sánchez-Valdivieso, 2018)

En la escala de Alvarado Modificado, se obtuvo, una sensibilidad del $69.78 \%$, especificidad $82.78 \%$, FVP $65.76 \%$ y FVN 20\%, para la escala de RIPASA una sensibilidad del $88.48 \%$, especificidad 90.9\%, FVP $68.9 \%$, FVN $25.58 \%$, con estos 
resultados de ambas escalas podemos decir que la escala de RIPASA presento una mejor sensibilidad y especificidad que la escala de Alvarado modificado. Resultados semejantes se obtuvieron por Guallpa Edison (2019), el cual trabajó con 201 pacientes en el Hospital José Carrasco Arteaga de Cuenca, donde se obtuvo una sensibilidad y especificidad del $98.34 \%$ y $75 \%$, respectivamente, en comparación con $93.92 \%$ y $85 \%$ para la escala de Alvarado Modificada (Guallpa Guallpa, 2019), demostrando que RIPASA lee mejor a los enfermos, pero Alvarado Modificado evalúa mejor a los sanos. (Guallpa Guallpa, 2019)

Shuaib A. (2016), estudio 134 pacientes, donde se halló en la escala de Alvarado Modificado una sensibilidad del $82.8 \%$ y especificidad 56\%; en la escala de RIPASA, (Almache Caiza \& Mena Chavarrea, 2019) sensibilidad del $94.5 \%$ y especificidad $88 \%$, llegando a la conclusión que la escala RIPASA es más preciso y específico en comparación a la escala de Alvarado modificado para la población de Kuwait. (Shuaib, et al., 2017)

C.Z.Díaz-Barrientos (2018), evaluó a 72 pacientes en el Hospital Universitario de Puebla, en el cual comparo las dos escalas y obtuvo como resultado una sensibilidad del $93.3 \%$ y especificidad $80.3 \%$ para la escala de RIPASA; una sensibilidad del $75 \%$ y especificidad 41.6\% para la escala de Alvarado Modificada (Arroyo-Rangel, Limon, Vera, Guardiola, \& Sánchez-Valdivieso, 2018), concluyendo que la escala de RIPASA comparada con la escala de Alvarado modificada no mostro ventajas al aplicarse a pacientes con sospecha de apendicitis aguda. (Almache Caiza \& Mena Chavarrea, 2019) (Díaz-Barrientos, y otros, 2018)

Reyes-Garcia et al. (2012), con 70 pacientes comparó las dos escalas en Hospital General de México hallando para la escala de Alvarado Modificado una sensibilidad del $89.5 \%$ y especificidad $69.2 \%$, en la escala de RIPASA una sensibilidad del $91.2 \%$ y especificidad de $84.6 \%$, concluyendo que ambas escalas presentaron buena sensibilidad para el diagnóstico de apendicitis aguda. Sin embargo, la escala de RIPASA presentó mejor especificidad y valores predictivos, con menor probabilidad de apendicectomías negativas. (Reyes-García, et al., 2012)

Nanjundaiah N. (2014), en la población india aplicó y comparó las dos escalas en 206 pacientes, donde encontró una sensibilidad del $96.2 \%$ y especificidad $90.5 \%$ para la escala de RIPASA, en la escala de Alvarado la sensibilidad fue del $58.9 \%$ y especificidad $85.7 \%$, señalando que la escala de RIPASA es un sistema de puntuación más conveniente, preciso y específico para poblaciones orientales que la escala de Alvarado. (Arroyo-Rangel, Limon, Vera, Guardiola, \& Sánchez-Valdivieso, 2018) (Nanjundaiah , Ashfaque , Venkatesh , Kalpana , \& Priya , 2014)

Pasumarthi, Vamsavardhan, y Madhu C. P. (2018), analizaron a 116 pacientes, donde aplicaron ambas escalas y encontraron una sensibilidad y especificidad de $52.08 \%$ y $80 \%$ respectivamente para la escala de Alvarado, en la escala de RIPASA la sensibilidad fue del $75 \%$, especificidad $65 \%$, indicando que la puntuación RIPASA es una herramienta de 
diagnóstico mucho mejor para el diagnóstico de apendicitis aguda. (Díaz-Barrientos C. , y otros, 2018) (Pasumarthi \& Madhu, 2018)

Maximos Frountzas (2018), realizó un meta-análisis en EEUU comparando las dos escalas RIPASA con Alvarado (estudio predecesor al de Alvarado Modificado) donde se evaluó a 2161 pacientes, encontrando sensibilidad para la escala de RIPASA del 94\% pero una especificidad significativamente de $55 \%$, indicando que la escala de RIPASA diferencia mejor a los enfermos. (Frountzas, y otros, 2018)

\section{Conclusiones.}

- El análisis exploratorio de datos manifiesta que el grado II de apendicitis (Apendicitis flegmonosa o fibrinosa) es la más frecuente en este análisis, mientras que los principales síntomas que ayudan a favorecer el diagnóstico de esta patología son el dolor migratorio $(71.5 \%)$ y el dolor en la fosa iliaca derecha (98\%), al mismo tiempo existen otros síntomas como la anorexia, náuseas o vómitos que en la personas observadas toman valores de $95.5 \%$ y $70 \%$ respectivamente (Almache Caiza \& Mena Chavarrea, 2019), en cuanto a las características biológicas el $50.8 \%$ corresponden a hombres y el $49.3 \%$ son mujeres, el $50 \%$ de los pacientes poseen una edad menor o igual a los 24 años,

- Para la construcción de la escala de Alvarado Modificada se considera 5 variables cualitativas (migración del dolor FID, anorexia, náuseas/vómitos, dolor en el cuadrante inferior derecho, signo de Bloomberg) y 3 variables cuantitativas (fiebre, leucocitos $>10.000 \mathrm{~mm}^{3}$, neutrofilia $>70 \%$ ); en relación con la escala de RIPASA se emplea 10 variables cualitativas (sexo, dolor fosa ilíaca derecha, náuseas/vómitos, dolor migratorio, anorexia, hipersensibilidad FID, resistencia muscular voluntaria, Rebote, Rovsing, examen general de orina negativo) y 4 variables cuantitativas (edad 40 años, síntomas $<48$ o $>48$, fiebre, leucocitos); cada una de las variables expuestas anteriormente en las dos escalas poseen un criterio de puntuación específico que al momento de ser sumados dichos valores permiten predecir el riesgo de padecer apendicitis.

- Existen 2 signos importantes que ayudan al diagnóstico correcto de apendicitis que son la hipersensibilidad en la fosa iliaca derecha y la resistencia muscular que toman valores de $97.75 \%$ y $91.25 \%$ respectivamente mientras que los signos como Rebote $(93.25 \%)$, Rovsing $(74.75 \%)$ y fiebre $\left(36^{\circ}\right)$ son detectados con gran frecuencia, pero no aportan significativamente al diagnóstico final. En los casos donde el juicio médico no se encuentre claro, es necesario acudir a algunos exámenes de laboratorio en esta investigación se toma en cuenta los leucocitos donde la mayoría de los pacientes asumen un conteo de 15.600 células $/ \mathrm{mm}^{3}$, neutrofilia con la mayor repetición del $84 \%$ y el examen general de orina en el cual el $85 \%$ de los pacientes obtuvieron un examen negativo. (Almache Caiza \& Mena Chavarrea, 2019)

- Finalmente, al contrastar la escala de Alvarado Modificada y RIPASA, se obtienen los siguientes resultados: Alvarado Modificada curva ROC (área 0.583), 
sensibilidad (69.78\%), especificidad (82.78\%), FVP (65.76\%), FVN (20\%), RIPASA curva ROC (área 0.594), sensibilidad (88.4\%), especificidad (90.9\%), FVP (68.90\%), FVN (25.58\%). Se concluye que la escala de RIPASA presenta mayor certeza al momento de diagnosticar apendicitis aguda, pero no existe una diferencia estadística muy marcada con respecto a la escala de Alvarado Modificada. (Almache Caiza \& Mena Chavarrea, 2019)

\section{Referencias bibliográficas.}

Almache Caiza, M., \& Mena Chavarrea, G. (2019). Comparación de la escala de alvarado modificada y la escala de ripasa a través de curvas ROC en el Hospital General Docente Riobamba, servicio de emergencia, junio 2010 - enero 2019. Obtenido de http://dspace.espoch.edu.ec/bitstream/123456789/13795/1/226T0053.pdf

Arroyo-Rangel, C., Limón, I. O., Vera, Á. G., Guardiola, P. M., \& Sánchez-Valdivieso, E. A. (2018). Sensitivity, specificity and reliability of the RIPASA Score for diagnosis of acute appendicitis in relation to the Alvarado Score. Cirugía Española, 96(3), 149-154.

Ávila, M., \& García-Acero, M. (2015). Apendicitis aguda: revisión de la presentación histopatológica en Boyacá, Colombia. Revista colombiana de Cirugía, 30(2), 125130.

Chong, C. F., Husain, S., Jumat, L., Chong, C. L., Tan , K. K., \& Yap , S. K. (2017). RIPASA Treatment Without Operation (TWO) - A Non-Inferiority Prospective Randomised Clinical Controlled Trial of Antibiotic Non-Operative Management Strategy versus Surgery Management Strategy for Early Uncomplicated Acute Appendicitis. Brunei Int Med J, 13(4), 111-123. Obtenido de http://www.bimjonline.com/PDF/Bimj\%202017\%20Volume\%2013,\%20Issue\% 204/BIMJ2017134111.pdf

Chong, C., Thien, A., Mackie, A., Tin, A., Tripathi, S., Ahmad, M., \& Telisinghe, P. (2011). Comparison of RIPASA and Alvarado scores for the diagnosis of acute appendicitis. Singapore medical journal, 52(5), 340.

Coursey, C., Nelson , R., Patel, M., Cochran, C., Dodd, L., DeLong, D., \& Vaslef, S. (2010). Making the diagnosis of acute appendicitis: do more preoperative CT scans mean fewer negative appendectomies? A 10-year study. Radiology, 254(2), 460-468.

de Quesada Suárez, L., Ival Pelayo, M., \& González Meriño, C. (2015). La escala de Alvarado como recurso clínico para el diagnóstico de la apendicitis aguda. Revista Cubana de Cirugía, 54(2), 121-128.

Díaz-Barrientos, C. Z., Aquino-González, A., Heredia-Montaño, M., Navarro-Tovar, F., Pineda-Espinosa, M. A., \& de Santillana, I. E. (2018). The RIPASA score for the diagnosis of acute appendicitis: A comparison with the modified Alvarado score. Revista de Gastroenterología de México, 83(2), 112-116. 
Díaz-Barrientos, C., Aquino-González, A., Heredia-Montaño, M., Navarro-Tovar, F., Pineda-Espinosa, M., \& de Santillana, I. (2018). Escala RIPASA para el diagnóstico de apendicitis aguda: comparación con la escala de Alvarado modificada. Revista de Gastroenterología de México,, 83(2), 112-116.

Fernández Zambrano, J. (2016). Apendicitis aguda, utilidad de la escala de Alvarado para su diagnóstico precoz, estudio a realizarse en el Hospital Teodoro Maldonado Carbo período 2015.

Fernández, J. (2016). Apendicitis aguda, utilidad de la escala de alvarado para su diagnóstico precoz.

Ferreira, G. (2019). Apendicitis aguda y Score de Alvarado, nuevo método diagnóstico. Universidad Nacional de Córdova. Obtenido de http://pa.bibdigital.uccor.edu.ar/1266/1/Apendicitis\%20aguda\%20y\%20score\%2 0de\%20Alvarado\%2C\%20nuevo\%20m\%C3\%A9todo\%20diagn\%C3\%B3stico.p df

Filiz, A., Aladag, H., Akin, M., Sucullu, I., Kurt, Y., Yucel, E., \& Uluutku, A. (2010). The role of d-lactate in differential diagnosis of acute appendicitis. Journal of Investigative Surgery, 23(4), 218-223.

Frountzas, M., Stergios, K., Kopsini, D., Schizas, D., Kontzoglou, K., \& Toutouzas, K. (2018). Alvarado or RIPASA score for diagnosis of acute appendicitis? A metaanalysis of randomized trials. International Journal of Surgery, 56, 307-314.

Golden , S. K., Harringa , J. B., Pickhardt , P. J., Ebinger , A., Svenson , J. E., Zhao , Y.Q., \& et al. (2016). Prospective evaluation of the ability of clinical scoring systems and physiciandetermined likelihood of appendicitis to obviate the need for CT. Emerg Med J, 33(7), 458-64.

Guallpa Guallpa, E. P. (2019). Validación de escalas RIPASA y Alvarado Modificada para diagnóstico de apendicitis aguda. Hospital Vicente Corral Moscoso y José Carrasco Arteaga. Cuenca 2017. UNIVERSIDAD DE CUENCA. Obtenido de http://dspace.ucuenca.edu.ec/bitstream/123456789/32094/1/Tesis.pdf

Humes, D., \& Simpson, J. (2012). Clinical presentation of acute appendicitis: clinical signs - laboratory findings - clinical scores, alvarado score and derivate scores. Imaging of acute appendicitis in adults and children. Springer, Berlin, Heidelberg, 13-21.

Klabtawee, W., Saensak, W., Khetsoongnern, A., \& Piriyasupong, T. (2011). Accuracy of RIPASA and modified RIPASA score comparing with Alvarado score for diagnosis of acute appendicitis and complication of acute appendicitis. Khon Kaen Medical Journal, 35(1), 38-47.

Laméris, W., Van Randen, A., Go, P., Bouma, W., Donkervoort, S., Bossuyt, P., \& Boermeester, M. (2009). Single and combined diagnostic value of clinical features and laboratory tests in acute appendicitis. Academic Emergency Medicine, 16(9), 835-842. 
López Abreu, Y., Fernández Gómez, A., Hernández Paneque, Y., \& Pérez Suárez, M. D. (2016). Evaluación de la Escala de Alvarado en el diagnóstico clínico de la apendicitis aguda. Revista Habanera de Ciencias Médicas, 15(2), 213-224.

Malik, M. U., Connelly, T. M., Awan, F., Pretorius, F., Fiuza-Castineira, C., El Faedy, O., \& Balfe, P. (2017). The RIPASA score is sensitive and specific for the diagnosis of acute appendicitis in a western population. International journal of colorectal disease, 32(4), 491-497.

Martin, M., \& Stella K, K. (2018). Acute appendicitis in adults: Diagnostic evaluation. Obtenido https://d1wqtxts1xzle7.cloudfront.net/40030840/Acute_appendicitis_in_adults_ _Diagnostic_evaluation.pdf?1447620698=\&response-contentdisposition=inline\%3B+filename\%3DAcute_appendicitis_in_adults_Diagnostic. pdf $\&$ Expires $=1618962602 \&$ Signature $=$ fDNfIROr1KRbTachM

Mendoza, J. D., Rodríguez, C. G., \& Guerrero, M. A. (2010). Evaluación prospectiva de la Escala de Alvarado en el diagnóstico de apendicitis aguda. Cirujano General, 32(1), 17-23.

Montero Tapia, E. (2016). Apendicectomías no enfermas en el Hospital Vicente Corral Moscoso y Hospital José Carrasco Arteaga, Cuenca 2014. Universidad de Cuenca. Obtenido de http://dspace.ucuenca.edu.ec/bitstream/123456789/23770/1/TESIS.pdf

Nanjundaiah, N., Ashfaque , M., Venkatesh, S., Kalpana , A., \& Priya , S. A. (2014). A Comparative Study of RIPASA Score and ALVARADO Score in the Diagnosis of Acute Appendicitis. Journal of Clinical and Diagnostic Research, NC03NC05. Obtenido de https://jcdr.net/article_fulltext.asp?issn=0973709 x $\&$ year $=2014 \&$ volume $=8 \&$ issue $=11 \&$ page $=$ NC03\&issn $=0973-$ $709 x \& \mathrm{id}=5170$

Orbea Marcial, V. H. (2012). Utilidad del Escala de Alvarado en el Diagnostico Temprano de Apendicitis Aguda; Hospital Provincial Puyo Abril-Diciembre 2009. 92-93. Escuela Superior Politécnica de Chimboraza.

Pasumarthi, V., \& Madhu, C. P. (2018). A comparative study of RIPASA score and ALVARADO score in diagnosis of acute appendicitis. International Surgery Journal, 5(3), 796-801.

Reyes-García, N., Zaldívar-Ramíre, F. R., Cruz-Martíne, R., Sandoval-Martíne, M. D., Gutiérrez-Banda, C. A., \& Athié-Gutiérrez, C. (2012). Precisión diagnóstica de la escala RIPASA para el diagnóstico de apendicitis aguda: análisis comparativo con la escala de Alvarado modificada. Cirujano General, 34(2), 101-106.

Sanabria, Á., Mora, M., Domínguez, L. C., Vega, V., \& Osorio, C. (2010). Validación de la escala diagnóstica de Alvarado en pacientes con dolor abdominal sugestivo de apendicitis en un centro de segundo nivel de complejidad. Revista Colombiana de Cirugía, 25(3), 195-201. 
Serrano Serrano , T. (2016). Valoración de la Escala de Alvarado como herramienta diagnóstica para apendicitis aguda comparado con el resultado Histopatológico en pacientes atendidos por el Servicio de Cirugía General en el Hospital General IESS Ambato en el período Junio 2015-Noviem. 2-4. Universidad Regional Autónoma de los Andes.

Serrano Serrano, T. C. (2016). Valoración de la Escala de Alvarado como herramienta diagnóstica para apendicitis aguda comparado con el resultado Histopatológico en pacientes atendidos por el Servicio de Cirugía General en el Hospital General IESS Ambato en el período Junio 2015-Noviem. 46. Universidad Regional Amazonica de los Andez.

Shrivastava, U., Gupta, A., \& Sharma, D. (2004). Evaluation of the Alvarado score in the diagnosis of acute appendicitis. Trop Gastroenterol, 25, 184-186.

Shuaib, A., Shuaib, A., Fakhra, Z., Marafi, B., Alsharaf, K., \& Behbehani, A. (2017). Evaluation of modified Alvarado scoring system and RIPASA scoring system as diagnostic tools of acute appendicitis. World journal of emergency medicine, 8(4), 276.

Smink, D., \& Soybel, I. (2019). Management of acute appendicitis in adults. Obtenido de https://www.uptodate.com/contents/management-ofacuteappendicitisinadults?search=porblema\%20apendicitis\%20aguda\&source $=\mathrm{s}$ earch_result\&selectedTitle=5 150\&usage_type=default\&display_rank=5

Thompson, N. A. (2012). Asociación entre Escala de Alvarado y diagnóstico de apendicitis aguda complicada y no complicada según anatomía patológica en el Centro Médico Naval. Horizonte Médico, 12(2), 14-20.

Thuijls, G., Derikx, J., Prakken, F., Huisman, B., van Bijnen Ing, A., van Heurn, E., \& Heineman, E. (2011). A pilot study on potential new plasma markers for diagnosis of acute appendicitis. The American journal of emergency medicine, 29(3), 256260.

Wani, M. M., Yousaf, M. N., Khan, M. A., BabaAbdul, A., Durrani, M., Wani, M. M., \& Shafi, M. (2007). Usefulness of the Alvarado scoring system with respect to age, sex and time of presentation, with regression analysis of individual parameters. Internet J Surg, 11(2), 1-5.

Zambrano, J., Ramos, D., \& Merino, R. (2019). Utilidad de la escala de alvarado en el diagnóstico precoz de apendicitis. Universidad Ciencia y Tecnología, 1(1), 7-7.

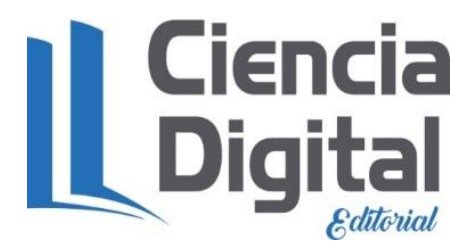


PARA CITAR EL ARTÍCULO INDEXADO.

Marcatoma Tixi, J. A., Mullo Guaminga, H. S., Pérez Londo, N. A., \& Almache Caiza, M. Y. (2021). Comparación de la Escala de RIPASA y Alvarado Modificada en la determinación de Apendicetomía a través de Curvas ROC . ConcienciaDigital, 4(2), 326345. https://doi.org/10.33262/concienciadigital.v4i2.1697

\section{¿Ciencia}

El artículo que se publica es de exclusiva responsabilidad de los autores y no necesariamente reflejan el pensamiento de la Revista Conciencia Digital.

El artículo queda en propiedad de la revista y, por tanto, su publicación parcial y/o total en otro medio tiene que ser autorizado por el director de la Revista Conciencia Digital.

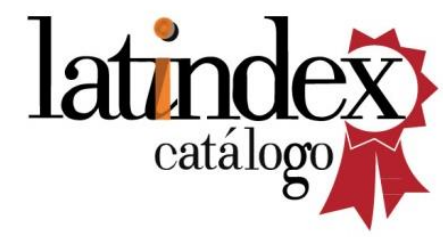

